\title{
Inclusive diffraction in DIS with the ZEUS detector
}

\author{
Marta Ruspa (on behalf of the ZEUS Collaboration) \\ Univ. Piemonte Orientale and INFN-Torino, Italy
}

\begin{abstract}
The diffractive dissociation of virtual photons, $\gamma^{\star} p \rightarrow X p$, has been studied with the ZEUS detector at HERA by requiring a large rapidity gap between $X$ and the outgoing proton, by analysing the mass distribution, $M_{X}$, of the hadronic final state, as well as by directly tagging the proton. At low values of the proton momentum loss, the diffractive structure functions extracted with the three methods agree, provided the different treatment and contributions of proton-dissociative events are taken into account.
\end{abstract}

\section{Inclusive diffraction at HERA}

In diffractive interactions in hadron-hadron or photon-hadron collisions at least one of the beam particles emerges intact from the collision, having lost only a small fraction of its initial energy, and carrying a small transverse momentum. Such interactions are described by the exchange of an object with vacuum quantum numbers, referred to as the Pomeron in the framework of Regge phenomenology [2]. Similar reactions can also proceed when quantum numbers are exchanged through subleading Reggeon and pion trajectories; however, these contributions are negligible at small values of the energy loss.

Significant progress has been made in understanding diffraction in terms of Quantum Chromo-Dynamics by studying the diffractive dissociation of virtual photons, $\gamma^{\star} p \rightarrow X p$, in deep inelastic ep scattering (DIS) at HERA (for a review see [3]). In this process, a photon of virtuality $Q^{2}$ diffractively dissociates interacting with the proton at a centre-ofmass energy $W$ and produces the hadronic system $X$ with mass $M_{X}$. The fraction of the proton's momentum carried by the exchanged object is denoted by $x_{\mathbb{P}}$, while the fraction of the momentum of the exchanged object carried by the struck quark is denoted by $\beta$.

\section{Comparison between selection methods}

Experimentally, diffractive $e p$ scattering is characterised by the presence of a leading proton in the final state carrying most of the proton beam energy and, consequently, by a lack of hadronic activity in the forward (proton) direction. Conservation of momentum implies that the system $X$ must have a small mass with respect to the photon-proton centre-ofmass energy, since $x_{\mathbb{P}} \gtrsim M_{X}^{2} / W^{2}$. These signatures have been widely exploited at HERA to select diffractive events by tagging the foward proton (proton-tagging method [4]), by requiring the presence of a large gap in the forward rapidity distribution of particles (LRG method [5]) or by exploting the shape of the $M_{X}$ distribution, different in diffractive and non-diffractive events $\left(M_{X}\right.$ method $\left.[6,7]\right)$.

A thorough comparison of these three selection methods has recently been carried out on a set of data collected with the ZEUS detector in the years 1999 and 2000 [8, 9], when the detector was still equipped with the leading proton spectrometer (LPS). The different methods access different kinematic regions and are subject to different systematic uncertainties: in the LRG and $M_{X}$ methods, high $M_{X}$ values are not accessible since the non-diffractive background grows with $M_{X}$ and the rapidity gap moves more and more forward (and is 
eventually confined to the beam pipe). Moreover, the measured cross section includes a contribution from events of the type $e p \rightarrow e X N$, in which the proton also dissociates into a state $N$ with low-mass $M_{N}$, separated from $X$ by a rapidity gap. The statistical precision of the results is good because of the high acceptance of the central detector. Conversely, low- $x_{\mathbb{P}}$ samples selected by the proton-tagging method have little or no background from proton-dissociative events or from non-diffractive DIS and allow access to higher values of $M_{X}$. However, the statistical precision is poor because of the small acceptance of the proton taggers - approximately $2 \%$ at low $x_{\mathbb{P}}$ in the LPS case.

In order to compare the reduced cross sections measured with the three methods, the different $x_{\mathbb{P}}$ and $M_{N}$ coverages of the various samples have to be taken into account:

- the LPS data extend up to $x_{\mathbb{P}}$ of 0.1 and therefore include contributions from Reggeon and pion trajectories; the LRG sample is restricted to the region $x_{\mathbb{P}}<0.02$ and thus mainly consists of diffractive events; in the $M_{X}$ sample, the statistical subtraction of the non-diffractive events has been shown [7] to suppress the Reggeon contribution;

- in the LPS results, $M_{N}$ coincides with the protons mass, $M_{p}$; the LRG data are also corrected to $M_{N}=M_{p}$; the $M_{X}$ results are corrected to $M_{N}<2.3 \mathrm{GeV}$.

The amount of proton-dissociation background and the corresponding corrections were found to be the most crucial issue in the comparison and are therefore discussed in detail in the following.

\subsection{Proton-dissociative background and relative corrections}

The proton-dissociative system can either escape entirely undetected in the forward beampipe or leak partially into the detector acceptance and therefore be measured by the forward detectors (forward plug calorimeter, FPC, and main calorimeter, CAL). In the former case, the background events are included in the measured cross section, of which they bias the normalisation. In the latter case, they are or are not rejected depending on the specific analysis cuts.

In the LPS analysis the contribution from proton-dissociative events was studied with the Pythia Monte Carlo (MC) and was found around $9 \%$ at $x_{\mathbb{P}}=0.1$, decreasing rapidly with decreasing $x_{\mathbb{P}}$. In the region $x_{\mathbb{P}}<0.02$ this background is negligible. At low $x_{\mathbb{P}}$ the ratio of the LRG and $M_{X}$ results to the LPS ones can thus be used to quantify the total fraction of proton-dissociative events included in these samples.

In the LRG analysis the contribution from proton-dissociative events was also estimated with Pythia. Two proton-dissociative samples were selected, one with the FPC and one with the LPS, the combination of which covers nearly the whole $M_{N}$ spectrum, including the lowest $M_{N}$ values. The generated PythiA distributions for $M_{N}, M_{X}$ and $Q^{2}$ were reweighted to describe these samples, in particular the energy distribution in the FPC and the $x_{L}$ distribution in the LPS. The average of the FPC and LPS estimates provided a measurement of the proton-dissociative contribution to the LRG results of $25 \pm 1$ (stat.) \pm 3 (syst.)\%.

In the $M_{X}$ method the diffractive contribution is extracted as the observed number of events after subtracting the non-diffractive component from a fit to the $\ln _{X}^{2}$ distribution. Proton-dissociative events measurable in the forward detectors lead to a reconstructed $M_{X}$ value higher than the actual photon-dissociative mass, hence to a distortion of the $\ln M_{X}^{2}$ spectrum and, consequently, of the extracted diffractive signal. The role and the treatment of the proton-dissociation background is thus much more critical than in the LRG analysis:

DIS 2008 
before the statistical subtraction of the non-diffractive background, all events need to be subtracted from the data which, according to a proton-dissociative MC, deposit anything measurable in the forward detectors. It has been shown [10] that, on average, events with masses $M_{N}<2.3 \mathrm{GeV}$ cannot be detected. The SANG MC was used to subtract events with $M_{N}>2.3 \mathrm{GeV}$ from the data. To avoid the Reggeon exchange region SANG, like most of the proton-dissociative MCs, is generated with an upper $M_{N}$ cut, $\left(M_{N} / W\right)^{2}<0.1$. Hence, bin-by-bin the amount of subtracted events with $M_{N}>2.3 \mathrm{GeV}$ depended on $W$. On the contrary, Monte Carlo studies showed that in the LRG case the rapidity gap requirement eliminates the $M_{N}$ tail; the correction becomes therefore independent of kinematics, as discussed in next Section and shown in Fig. 1.

\section{Comparison between cross section results}

The results obtained with the three methods were compared in bins of $M_{X}, Q^{2}$ and $x_{\mathbb{P}}$ in terms of the diffractive reduced cross section, $\sigma_{r}^{D(3)}$. The latter coincides with the diffractive structure function, $F_{2}^{D(3)}$, if the ratio of the cross sections for longitudinally and transversely polarised virtual photons can be neglected. The three samples are only weakly correlated through systematics but statistically not independent: the LRG and $M_{X}$ data overlap by about $75 \% ; 0.7 \%$ of the LRG events have a proton measured in the LPS and $35 \%$ of the LPS events are also contained in the LRG sample.

The ratio of $\sigma_{r}^{D(3)}$, extracted from the LPS and LRG data, shown in Fig. 1, is $0.76 \pm$

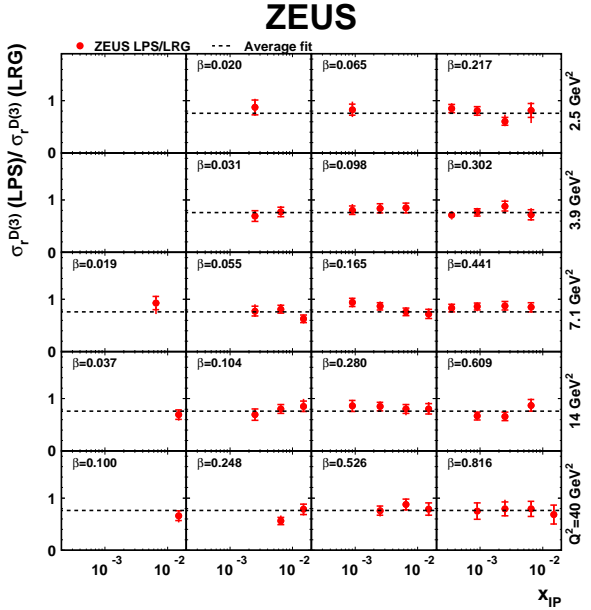

Figure 1: Ratio LPS/LRG. 0.01 (stat.) ${ }_{-0.02}^{+0.03}$ (syst.) ${ }_{-0.05}^{+0.08}$ (norm.); the last uncertainty reflects the normalisation uncertainty of the LPS data, mostly related to the $\pm 7 \%$ uncertainty due to the proton-beam optics. The ratio is independent of $Q^{2}, x_{\mathbb{P}}$ and $\beta$, indicating that the two methods lead to compatible results for $x_{\mathbb{P}}<0.01$. It also confirms that contributions from proton-dissociative events in the LRG measurement do not significantly alter the $Q^{2}, x_{\mathbb{P}}$ or $\beta$ dependences. The ratio translates into a proton-dissociative background fraction of $24 \pm 1$ (stat. $)_{-3}^{+2}$ (syst.) $)_{-8}^{+5}$ (norm.)\%. The agreement between this number and the result of the MC study discussed in Sec. 2.1 lends support to the present estimate of the proton- dissociation contamination in the LRG analysis.

Cross section measurements obtained with the LRG and $M_{X}$ methods are compared in Fig. 2, where also the previous $M_{X}$-method results [7] are shown. The LRG data were corrected to $M_{N}=M_{p}$ by statistical subtraction of the background estimated in Sec. 2.1. The $M_{X}$ results were normalised to the LRG data with a scaling factor of $0.83 \pm 0.04$, obtained from a global fit; this factor quantifies the amount of residual proton-dissociative background in the $M_{X}$ data due to masses below $2.3 \mathrm{GeV}$. The overall agreement between the two measurements is satisfactory. The different $x_{\mathbb{P}}$ dependence for $x_{\mathbb{P}} \gtrsim 0.01$, more evident at low $Q^{2}$, may be ascribed to the fact that in the $M_{X}$ results the contribution of Reggeon and pion trajectories is suppressed. In the low- $Q^{2}$ region, the $Q^{2}$ behaviour is 
slightly different in the two data sets, with the $M_{X}$-method results decreasing faster with $Q^{2}$ than the LRG results.

ZEUS

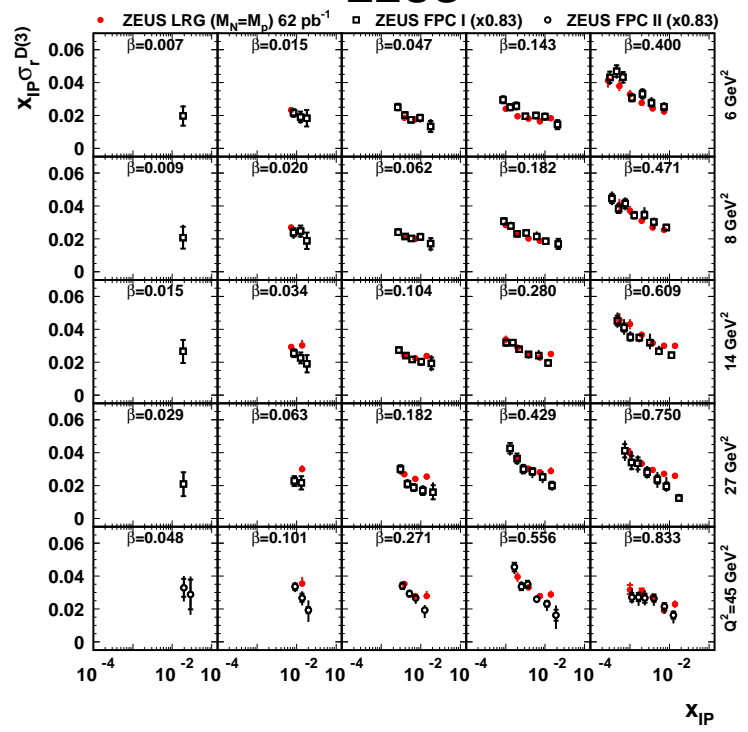

ZEUS

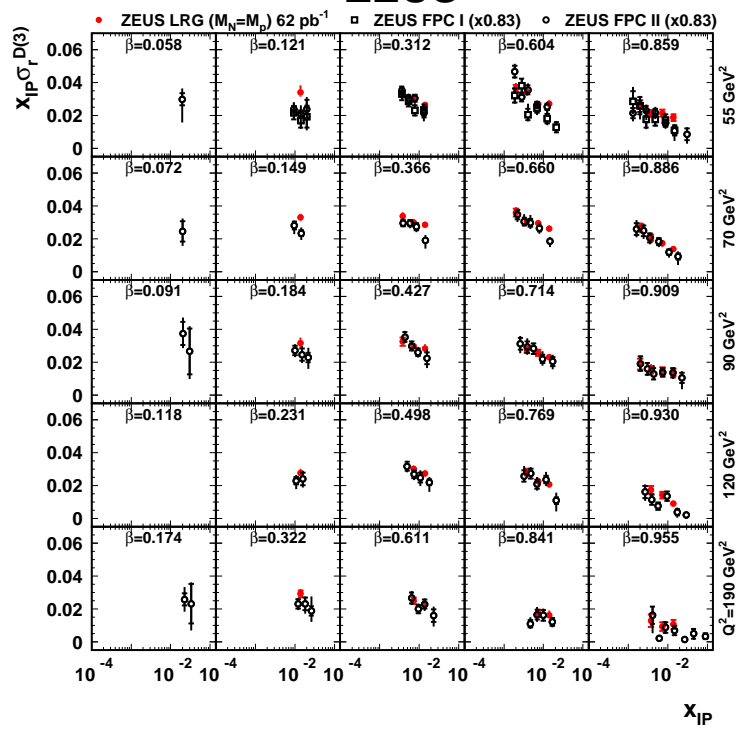

Figure 2: Comparison $M_{X}$-LRG.

\section{References}

[1] Slides:

http: //indico. cern. ch/contributionDisplay $\cdot$ py? contribId=23\&sessionId=16\& conf Id=24657

[2] P.D.B. Collins, An Introduction to Regge Theory and High Energy Physics, Cambridge University Press, Cambridge (1977).

[3] M. Arneodo and M. Diehl, Diffraction for non believers, arXiv:hep-ph/0511047 (2005).

[4] H1 Coll., A. Aktas et al., Eur. Phys. J. C48 749 (2006); ZEUS Coll., S. Chekanov et al., Eur. Phys. J. C38 43 (2004); ZEUS Coll., S. Chekanov et al., Eur. Phys. J. C25 169 (2002); ZEUS Coll., J. Breitweg et al., Eur. Phys. J. C1 81 (1997).

[5] H1 Coll., A. Aktas et al., Eur. Phys. J. C48 715 (2006); H. Abramowicz, Int. J. Mod. Phys. A 15 S1, 495 (2000).

[6] http://www-h1.desy.de/h1/www/publications/H1_sci_results.shtml, H1-prelim06-014; ZEUS Coll., S. Chekanov et al., Eur. Phys. J. C25 169 (2002).

[7] ZEUS Coll., S. Chekanov et al., Nucl. Phys. B713, 3 (2005).

[8] ZEUS Coll., S. Chekanov et al., arXiv:hep-ex/0802.3017v2, accepted by Nucl. Phys. B.

[9] http://www-zeus.desy.de/public_results/groupsearch.php, ZEUS-pub-08-010.

[10] H. Lim, Ph.D. Thesis, The Graduate School, Kyungpook National University, Taegu (Republic of Korea), unpublished (2002). 